\title{
Determinantes probabilísticos del riesgo de deserción en las Universidades de Nariño y del Valle (Colombia) a partir de los registros establecidos por el Ministerio de Educación Nacional (2007-2017)
}

\section{Probabilistic determinants of the risk of desertion in the Universities of Nariño and the Valle (Colombia) from the records established by the ministry of national education (2007-2017)}

DOI: http://dx.doi.org/10.17981/cultedusoc.11.1.2020.08

Recibido: 23/10/2019. Aceptado: 19/02/2020. Publicado: 12/03/2020

Marco Antonio Burgos Flórez

Universidad de Nariño. Pasto (Colombia) marcoantonioburgos@gmail.com

Katherin Julieth Ruales Suárez

Universidad de Nariño. Pasto (Colombia) katherin2320@hotmail.com

Yhony Estivel Bastidas García

Universidad de Nariño. Pasto (Colombia) yhobas1991@gmail.com

Carlos Manuel Córdoba Segovia

Universidad de Nariño. Pasto (Colombia) carcord13@yahoo.es

Para citar este artículo:

Burgos, M., Ruales, K., Bastidas, Y. y Córdoba, C. (2020). Determinantes probabilísticos del riesgo de deserción en las Universidades de Nariño y del Valle (Colombia) a partir de los registros establecidos por el Ministerio de Educación Nacional (2007-2017). Cultura, Educación y Sociedad, 11(1). 117-126. DOI: http://dx.doi.org/10.17981/cultedusoc.11.1.2020.08

\section{Resumen}

En el artículo se analizan los principales determinantes probabilísticos del riesgo de deserción en la población estudiantil de las Universidades de Nariño y del Valle (Colombia), a partir de la clasificación hecha por el Sistema para la Prevención de la Deserción en las Instituciones de Educación Superior-Spadies, con riesgo de abandono medio y medio alto en la escala valorativa. El fundamento metodológico es analítico parte de la estimación de un modelo probit, con variable discreta dependiente dicotómica "grupo de riesgo" y varias regresoras continuas y discretas. En los resultados se determina el perfil del estudiante con mayor riesgo de deserción en las dos universidades representado por sujetos hombres adscritos a la Facultad de Ciencias Exactas y Naturales, que no disponen de apoyos financieros dentro de la universidad donde se adscriben. Se concluye que la deserción, además de afectar al estudiante como ser social, incide en forma negativa en el desempeño de las instituciones de educación superior por su corresponsabilidad en la formación de un ciudadano integral.

Palabras clave: Riesgo de deserción; educación; universidades; modelos probabilísticos.

\section{Abstract}

The article analyzes the main probabilistic determinants of dropout risk in the student population of the Universities of Nariño and del Valle, Colombia, based on the classification made by the System for the Prevention of Attrition in Higher Education Institutions ( Spadies), with medium and medium high abandonment risk on the rating scale. The analytical methodological basis is based on the estimation of a probit model, with a dichotomous dependent discrete variable "risk group" and several continuous and discrete return. The results determine the profile of the student with the highest risk of dropping out in the two universities represented by male subjects assigned to the Faculty of Exact and Natural Sciences, who do not have financial support within the university where they are assigned. It is concluded that attrition, in addition to affecting the student as a social being, has a negative impact on the performance of higher education institutions due to their co-responsibility in the formation of an integral citizen.

Keywords: Attrition risk; education; universities; probabilistic models. 


\section{INTRODUCCIÓN}

El presente artículo es resultado de un proyecto de investigación denominado "Diagnóstico de la población estudiantil de la Universidad de Nariño con riesgo de deserción, sede Pasto, 2018", financiada por el Sistema de Investigaciones de la Universidad de Nariño en la convocatoria docente de investigación del año 2018.

Se parte de la premisa que actualmente la educación es considerada una de las herramientas fundamentales para lograr el desarrollo económico y social de las regiones y países; específicamente, la educación superior constituye un elemento clave para la consolidación de procesos de desarrollo sostenible y sustentable en medio de un mundo de globalización y modernización permanente. No obstante, el acceso a la educación superior se ha convertido en un reto para miles de personas a nivel mundial, particularmente en países en vías de desarrollo como Colombia, en donde los obstáculos de adhesión se incrementan a medida que la vulnerabilidad socio-económica del aspirante también lo hace, menoscabando de esa forma el desarrollo humano integral particularmente, de aquellas regiones que se caracterizan por una baja cobertura de la educación superior pública.

La aparición y el desarrollo de la deserción estudiantil ha generado grandes consecuencias de orden social y económico, representa un importante problema del sistema educativo nacional; las elevadas tasas de abandono de los estudios conllevan importantes situaciones, además, de afectar al estudiante y su rol dentro de la sociedad, incide negativamente en la Institución de Educación Superior-IES, a la que se encuentra adscrito puesto que, si bien, la responsabilidad fundamental de la universidad es formar a los futuros profesionales, esta debe garantizar los medios indispensables para que el estudiante pueda culminar dicha etapa, brindándole un acompañamiento integral y permanente.

Por lo anterior, el presente artículo de investigación aborda, en primer lugar, los principales referentes teóricos que permiten comprender y abordar el fenómeno de una manera científica; en segundo lugar, la metodología de la investigación, luego los resultados y finalmente las conclusiones del estudio.

\section{REFERENTES TEÓRICOS}

El tema de deserción estudiantil ha sido centro de diversas investigaciones tanto a nivel internacional como nacional; no obstante, es evidente que en los países con menor grado de desarrollo, el problema de deserción es un común denominador, por lo cual el número de estudios alrededor del tema es mucho mayor que los trabajos encontrados en países desarrollados; aun así, el riesgo de deserción es una temática poco conocida y estudiada, suscitando la premisa de que generalmente se analiza el problema una vez ocurrido y no con el propósito de prevención.

El riesgo de deserción está determinado como la probabilidad que presenta un estudiante perteneciente al Sistema de Educación Superior nacional de abandonar sus estudios por más de dos períodos consecutivos o, en otras palabras, la probabilidad de desertar, sea del programa en el que se encuentra matriculado, de la Institución de Educación Superior, y/o del sistema educativo en general. 
Berrio, Misas, Santacruz y Villa (2013) mencionan como la deserción estudiantil al ser uno de los problemas que inciden, de manera importante, sobre la formación de capital humano, siendo éste un factor determinante para el crecimiento económico y, por ende, para el incremento en la calidad de vida de la población; un hecho relevante, sobre todo, en países en desarrollo como Colombia, donde el proceso de acumulación de capital humano es aún bajo.

No obstante, autores como Tinto citado por Ministerio de Educación Nacional-MEN (2009, p. 20), afirman que el estudio de la deserción en la educación superior es extremadamente complejo, ya que implica, no solamente, una variedad de perspectivas sino, también, una gama de diferentes tipos de abandono. Adicionalmente, sostiene que ninguna definición puede captar en su totalidad la complejidad de este fenómeno, quedando en manos de los investigadores la elección de la aproximación que mejor se ajuste a sus objetivos y al problema por investigar.

El abandono del sistema educativo por parte de un individuo genera una problemática con varias aristas; por una parte, desde el punto de vista de la institución se produce un efecto en los ingresos de la misma, teniendo en cuenta que los costos de los derechos académicos de los estudiantes se constituyen en los ingresos de las instituciones prestadoras del servicio de educación.

Por otra parte, también existe un efecto en la situación familiar de la persona, puesto que una mayor cualificación permite a los individuos acceder a mejores remuneraciones salariales en la medida en que se incrementa la productividad. El efecto también se refleja en las mejores condiciones socioeconómicas a las cuales podría acceder la persona si continuara con su proceso educativo. El efecto directo se ve a partir del abandono puesto que, para llegar a ese momento, se ha tenido que recurrir a una serie de flujos de caja negativos, que se pierden cuando la meta no se logra. Este se vuelve más perjudicial cuando se trata de una deserción tardía. Desde esta perspectiva, es necesario que el individuo desarrolle estrategias emocionales que permitan el reforzamiento y la motivación para salir adelante. (Akl, Jiménez y Aponte, 2016)

A partir de los estudios de Tinto en 1982 y de Giovagnoli en 2002, el MEN (2008, p. 18) conceptualiza la deserción como una situación enfrentada por un estudiante cuando aspira y no logra concluir su proyecto educativo, considerando como desertor al individuo que siendo estudiante de una institución de educación superior no presenta actividad académica por dos semestres consecutivos, lo cual equivale a un año de inactividad académica.

El riesgo de deserción, en ese sentido, es un cálculo importante en la medida que permite establecer qué tan propenso es un individuo a abandonar sus estudios y debería, por tanto, articularse con las distintas políticas establecidas por el MEN en general y, por las IES en particular, en pro de la retención de los individuos con alto riesgo.

A pesar de que las variables determinantes para el cálculo del riesgo están enmarcadas en los ámbitos individual, académico, institucional y socioeconómico, el MEN no establece para conocimiento de la comunidad la metodología utilizada para dicho cálculo, por lo cual este estudio se soporta en determinar, a partir de las variables establecidas en los reportes de riesgo de deserción enviados a cada IES, discriminados para cada estudiante por parte del SPADIES. La probabilidad que tienen los estudiantes de estar en categoría media y media alta de riesgo de desertar está en función de variables como género, rango 
de ingreso familiar, posesión de vivienda propia, el nivel educativo de la madre, puntaje obtenido en las pruebas Saber 11 realizadas por el Instituto Colombiano para la Evaluación de la Educación-ICFES y la respectiva edad de presentación a dichas pruebas, recepción de apoyos financieros y de Icetex, Cohorte y Facultad a la que pertenece.

\section{Metodología}

Para efectos de la presente investigación, se clasifica al fenómeno del riesgo de deserción como un problema de carácter discreto y no como uno continuo, teniendo en cuenta que se desea modelar situaciones en donde existe la probabilidad de que ocurra o no determinado fenómeno que, como en el presente caso, es el de caer en riesgo de desertar de la educación superior en una universidad de carácter público.

El modelo clásico de regresión no otorga la mejor determinación para el problema estudiado, por lo cual se hace necesario considerar modelos de respuesta binaria con mayor grado de sofisticación, la cual incluye estimaciones acordes a los modelos probabilísticos, particularmente al modelo probit. De acuerdo con Stock y Watson (2012), las regresiones de tipo probit son modelos de carácter no lineal diseñados específicamente para variables dependientes binarias.

Debido a que una regresión con una variable dependiente binaria Y modeliza la probabilidad de que $\mathrm{Y}=1$, tiene sentido adoptar una formulación no lineal que obligue a que los valores estimados estén entre 0 y 1 . Debido a que las funciones de distribución de probabilidad acumuladas (f.d.a.) dan lugar a probabilidades entre 0 y 1 , éstas se utilizan en las regresiones logit y probit.

La regresión probit utiliza la f.d.a. normal estándar (p. 279).

El modelo probit con varios regresores es calculado para explicar el z-valor y está representado mediante la fórmula 1.

$$
\operatorname{Pr}\left(Y=1 \mid X_{1} X_{2}\right)=\phi\left(\beta_{0}+\beta_{1} X_{1}+\beta_{2} X_{2}+\cdots+\beta_{k} X_{k}\right)
$$

Donde $Y$ es la variable dependiente dicotómica. $B$ es el coeficiente que adopta determinado regresor $X$. $\Phi$ es la función de distribución normal estándar acumulada.

Entonces, el z-valor está dado por el valor obtenido en la fórmula 2 buscado en la tabla de distribución normal:

$$
z=\beta_{0}+\beta_{1} X_{1}+\beta_{2} X_{2}+\cdots+\beta_{k} X_{k}
$$

Generalmente, el efecto sobre $Y$ de un cambio en $X$ es el cambio esperado en $Y$ que surge de un cambio de $X$, expresado en $\beta$. "Cuando $Y$ es binaria, su esperanza condicional es la probabilidad condicional de que sea igual a 1, por lo que el cambio esperado en $Y$ que surge de un cambio en $X$ es el cambio en la probabilidad de que $Y=1$ " (Stock y Watson, 2012, p. 281).

Según Méndez (s.f.), conocer la distribución de la probabilidad de los estadísticos, es un hecho que posibilita obtener conclusiones a partir de una muestra hacia la población en general, proporcionar una medida del error que puede surgir en dichos cálculos y conclusiones y dar una medida de confianza de que ese sea el error y no uno mayor. 
Considerando que el supuesto de los modelos probit es el de la normalidad de los errores muestrales, hay que tener en cuenta el teorema del límite central, que especifica la regularidad estadística de las medias aritméticas:

[..] es uno de los resultados fundamentales de la estadística. Este teorema afirma que si una muestra es lo bastante grande (generalmente cuando el tamaño muestral (n) supera los 30), sea cual sea la distribución de la media muestral, seguirá aproximadamente una distribución normal. Es decir, dada cualquier variable aleatoria, si se extraen muestras de tamaño $n(n>30)$ y se calculan los promedios muestrales, dichos promedios seguirán una distribución normal. Además, la media será la misma que la de la variable de interés, y la desviación estándar de la media muestral será aproximadamente el error estándar (Canal, 2006, p. 118).

Con objeto de estimar cuál es la probabilidad de un estudiante de la Universidad de Nariño caer en riesgo de deserción, se tienen en cuenta ciertas variables que permiten explicar en parte la consolidación de dicho fenómeno y además se procede a realizar la misma estimación para la UniValle (Anexos A, B, C, D, E y F), considerandola como la universidad pública acreditada en Alta Calidad de mayor representación en la Zona Pacífico de Colombia, con el propósito de establecer un comparativo que posibilite identificar la condición en la que se encuentran los estudiantes de la Universidad de Nariño, aun en su condición de IES de carácter regional.

\section{REsultados}

Las variables más determinantes en la probabilidad de caer en riesgo de deserción se encuentran relacionadas en la Tabla 1 para cada universidad.

TABLA 1.

Variables determinantes del riesgo de deserción. Universidades de Nariño y del Valle. 2007-2017

\begin{tabular}{llll}
\hline & & & Universidad del Valle \\
\hline Variable & Tipo & Variable & Tipo \\
& $\begin{array}{l}\text { Dependiente } \\
\text { Grupo de Riesgo }\end{array}$ & Grupo de Riesgo & Dependiente \\
& $\begin{array}{l}\text { Binaria } \\
\text { Dicotómica }\end{array}$ & & Binaria \\
Dicotómica \\
Facultad de Artes & Discreta & Facultad de Artes Integradas & Discreta \\
Facultad de Ciencias Agrícolas & Discreta & Facultad de Ciencias Naturales y Exactas & Discreta \\
Facultad de Ciencias de la Salud & Discreta & Facultad de Ciencias de Administración & Discreta \\
Facultad de Ciencias Exactas y Naturales & Discreta & Facultad de Salud & Discreta \\
Facultad de Ingeniería & Discreta & Facultad de Humanidades & Discreta \\
Facultad de Agroindustrial & Discreta & Facultad de Ingeniería & Discreta \\
Ingreso Familiar entre 2 y 7 salarios & Discreta & Cohorte & Continua \\
Mujer & Discreta & Mujer & Discreta \\
Puntaje Icfes & Continua & Puntaje Icfes & Continua \\
Educación de la Madre: Primaria & Discreta & Educación de la Madre: Primaria & Discreta \\
Edad de Presentación de Icfes & Continua & Edad de Presentación de Icfes & Continua \\
Posee vivienda propia & Discreta & Posee vivienda propia & Discreta \\
No ha obtenido apoyo financiero & Discreta & No ha obtenido apoyo financiero & Discreta \\
& & No ha obtenido apoyo de Icetex & Discreta \\
\hline
\end{tabular}

Fuente: Elaboración propia con base en información suministrada por el sistema SPADIES (MEN, 2019). 
Las variables discretas son aquellas que toman valores de 1 cuando el estudiante tiene dicho atributo y 0 de lo contrario. Las variables continuas toman cualquier valor, no están condicionadas a ubicarse entre 0 y 1.

En cuanto a la variable dependiente discreta dicotómica "grupo de riesgo", toman valores de 1 para las dos Universidades, cuando la clasificación hecha por Spadies para el estudiante se encuentra entre Riesgo Medio y Medio Alto y valores de 0 cuando el grupo se ubica entre Riesgo Bajo y Medio Bajo. Los resultados obtenidos a partir de los modelos probabilísticos se muestran en la Tabla 2.

TABLA 2.

Resultados de modelos probabilísticos con variable dependiente dicotómica grupo de riesgo, Universidades de Nariño y del Valle. 2007-2017

\begin{tabular}{|c|c|c|c|c|c|}
\hline \multicolumn{3}{|c|}{ Universidad de Nariño } & \multicolumn{3}{|c|}{ Universidad del Valle } \\
\hline Variable & MFX & $\begin{array}{l}\text { Coef. } \\
\text { MFX }\end{array}$ & Variable & MFX & $\begin{array}{l}\text { Coef. } \\
\text { MFX }\end{array}$ \\
\hline Facultad de Artes & & $4,78 \%$ & Facultad de Artes Integradas & & $-1,33 \%$ \\
\hline Facultad de Ciencias Agrícolas & & $11,79 \%$ & $\begin{array}{l}\text { Facultad de Ciencias Naturales y } \\
\text { Exactas }\end{array}$ & & $10,29 \%$ \\
\hline Facultad de Ciencias de la Salud & & $-7,76 \%$ & $\begin{array}{l}\text { Facultad de Ciencias de } \\
\text { Administración }\end{array}$ & & $-1,77 \%$ \\
\hline $\begin{array}{l}\text { Facultad de Ciencias Exactas y } \\
\text { Naturales }\end{array}$ & & $13,65 \%$ & Facultad de Salud & & $-6,26 \%$ \\
\hline Facultad de Ingeniería & & $19,27 \%$ & Facultad de Humanidades & & $-2,10 \%$ \\
\hline $\begin{array}{l}\text { Facultad de Ingeniería } \\
\text { Agroindustrial }\end{array}$ & & $12,34 \%$ & Facultad de Ingeniería & & $6,82 \%$ \\
\hline $\begin{array}{l}\text { Ingreso Familiar entre } 2 \text { y } 7 \\
\text { Salarios }\end{array}$ & $15,30 \%$ & $10,21 \%$ & Cohorte & $16,93 \%$ & $0,50 \%$ \\
\hline Mujer & & $-9,52 \%$ & Mujer & & $-12,34 \%$ \\
\hline Puntaje Icfes & & $-0,25 \%$ & Puntaje Icfes & & $-0,29 \%$ \\
\hline Educación de la Madre: Primaria & & $-2,22 \%$ & $\begin{array}{l}\text { Educación de la Madre: } \\
\text { Primaria }\end{array}$ & & $-3,63 \%$ \\
\hline Edad de Presentación de Icfes & & $1,10 \%$ & Edad de Presentación de Icfes & & $1,11 \%$ \\
\hline Posee vivienda propia & & $-3,00 \%$ & Posee vivienda propia & & $-2,43 \%$ \\
\hline \multirow[t]{2}{*}{ No ha obtenido apoyo financiero } & & $11,53 \%$ & No ha obtenido apoyo financiero & & $18,71 \%$ \\
\hline & & & No ha obtenido apoyo de Icetex & & $2,01 \%$ \\
\hline
\end{tabular}

Fuente: Elaboración propia.

La probabilidad que tiene un estudiante de la Universidad de Nariño de entrar en riesgo de deserción presentando todas las características atribuidas por el modelo es de 15,30\% y para un estudiante de la Universidad del Valle es de $16,93 \%$.

En cuanto a la Universidad de Nariño es posible mencionar que pertenecer a las Facultades de: Ingeniería, Ciencias Exactas y Naturales, Ingeniería Agroindustrial y Ciencias Agrícolas, incrementa la probabilidad de riesgo de deserción en 19,27\%, 13,65\%, 12,34\% y 11,79\%, respectivamente, mientras que pertenecer a la Facultad de Ciencias de la Salud la disminuye en $7,76 \%$. 
En la Universidad del Valle, pertenecer a la Facultad de Ciencias Exactas y Naturales incrementa la probabilidad de riesgo de deserción en 10,29\%, mientras que pertenecer a las Facultades de: Artes Integradas, Administración, Humanidades, Facultad de Salud e Ingeniería, la reduce en $1,33 \%, 1,77 \%, 2,10 \%, 6,26 \%$ y $6,82 \%$, respectivamente.

Se observa entonces que las dos universidades cuentan con estudiantes con alto riesgo de desertar en la Facultad de Ciencias Exactas y Naturales, no obstante, en la Facultad de Artes se presenta un comportamiento inverso. Ser mujer reduce la probabilidad de caer en riesgo de deserción en 9,52\% en la Universidad de Nariño y un 12,34\% en la Universidad del Valle.

En cuanto a la variable ingresos, en la Universidad de Nariño se encontró que, en promedio, a partir del año 2007, el 58,76\% de la población estudiantil tiene ingresos familiares ubicados entre los 2 y los 7 SMMLV, específicamente entre los 2 y 3 salarios, ingresos con los que el $87 \%$ de los hogares deben atender las necesidades de 2 a 5 personas que lo integran. Por ello, la variable ingresos familiares, cuando se ubica en dicho rango, incrementa en un 10,21\% la probabilidad de caer en riesgo de deserción, al considerar que la falta de ingresos limita el completo desarrollo de las actividades académicas, sea por la necesidad de trabajar que tiene el estudiante o por la falta de recursos para poder adquirir materiales de estudio, buena alimentación, transporte, etc. En la Universidad del Valle, la variable ingresos no resultó significativa.

No obtener apoyo financiero aporta un incremento sustancial a la hora de caer en riesgo de deserción por parte de los estudiantes de ambas universidades, 11,53\% en la Universidad de Nariño y 18,71\% en la del Valle. Aun cuando en esta última, la variable ingresos familiares no es significativa, es posible observar que el factor económico tiene gran influencia en el fenómeno de deserción, particularmente de las universidades públicas, puesto que a partir de este factor se da el sostenimiento del estudiante y tener ingresos bajos, limita su proceso formativo.

\section{CONCLUSIONES}

La educación se constituye como eje fundamental para lograr el desarrollo de las personas y las sociedades, pues además de participar en la formación de seres humanos de manera integral, es clave en el crecimiento económico de las regiones y en la consecución de su bienestar. No obstante, problemas como la baja cobertura de educación superior en Colombia, el fenómeno de la deserción estudiantil, los problemas de financiamiento, etc., han generado grandes consecuencias de orden social y económico y se constituyen como un importante problema del sistema educativo nacional y de las universidades.

El fenómeno de deserción estudiantil, en particular, además de afectar al estudiante, su rol dentro de la sociedad y su calidad de vida futura, incide de manera negativa en el desempeño de las instituciones de educación superior, pues es su responsabilidad brindar apoyo a todos aquellos que presentan vulnerabilidad, sea de tipo académico, económico, social o emocional. Por otro lado, la deserción estudiantil afecta la formación de capital humano, concretamente en Colombia, donde el proceso de acumulación de capital humano es aún bajo, aun cuando este es un factor determinante para el crecimiento económico. 
Entre las principales causas del fenómeno, el MEN ha ideado un estado del arte que incluye determinantes de carácter individual como edad, género, entorno familiar, estado o civil, etc.; académicos como orientación profesional, rendimiento académico, calidad del programa al que está adscrito, entre otros; institucionales como recursos universitarios o normalidad académica y socioeconómicos como estrato, ingresos, situación laboral, dependencia económica, etc. De ellos, el género, la orientación profesional en los colegios o mala elección de la carrera, la situación económica y el acceso a financiamiento o becas, son los determinantes que más influyen en el fenómeno en las universidades del país.

Los resultados específicos de la investigación permiten establecer que la probabilidad de riesgo de deserción es más alta en la Universidad del Valle cuando se toman en conjunto todas las variables analizadas en el modelo, ubicándose en casi el 17\% de probabilidad, frente a un $15,30 \%$ presentado por la Universidad de Nariño con las características atribuidas en su modelación econométrica. No obstante, es posible mencionar que como común denominador para las dos universidades, un estudiante con riesgo medio y medio alto de desertar es aquel que pertenece a la Facultad de ciencias exactas y naturales, es hombre y no tiene apoyo financiero por parte de la IES.

El fenómeno de la deserción resulta ser multicausal y es tarea de las IES y del MEN, prevenirlo.

\section{REFERENCIAS}

Akl, P. M., Jiménez, E. P. y Aponte, F. L. (2016). Estrategias de afrontamiento en mujeres víctimas de violencia intrafamiliar. Cultura Educación y Sociedad, 7(2), 105-121. Recuperado de https://revistascientificas.cuc.edu.co/culturaeducacionysociedad/article/view/1105

Berrío, M., Misas, M., Santacruz, S. y Villa, E. (2013). Análisis de deserción estudiantil en la Pontificia Universidad Javeriana-Bogotá: Caracterización de la población estudiantil. Vniversitas Económica, (7), 1-59. Recuperado de http://cea.javeriana.edu.co/documents/153049/2786252/Vol.13_7_2013.pdf/bef56a7c-5817-413d-9485-7613a2cc93cb

Canal, N. (2006). Distribuciones de probabilidad. El teorema central del límite. Revista Seden, 107-120. Obtenido de https://www.revistaseden.org/files/8-CAP\%208.pdf

Méndez, I. (s.f.). Teorema de Límite Central. Valoración estadística en la investigación. [Online]. Obtenido de http://fcm.ens.uabc.mx/ chelo/estadistica/doc-pdf/t-l-c.pdf

República de Colombia. MEN. (2019). SPADIES. [Online]. Disponible en https://www.mineducacion.gov.co/1621/article-156292.html

República de Colombia. MEN. (2009). Deserción estudiantil en la educación superior Colombiana: Metodología de seguimiento, diagnóstico y elementos para su prevención. [Online]. Obtenido de http://www.mineducacion.gov.co/sistemasdeinformacion/1735/ articles-254702_libro_desercion.pdf

República de Colombia. MEN. (2008). Deserción estudiantil en la educación superior colombiana, elementos para su diagnóstico y tratamiento. [Online]. Obtenido de http:// www.mineducacion.gov.co/sistemasdeinformacion/1735/articles-254702_diagnostico_ desercion.pdf

Stock, J. H. y Watson, M. M. (2012). Introducción a la Econometría (3 Ed.) Madrid: Pearson. 


\section{Anexos}

Anexo A.

Determinantes probabilísticos del riesgo de deserción en la Universidad del Valle a partir del uso de modelos Probit. 2007 - 2017.

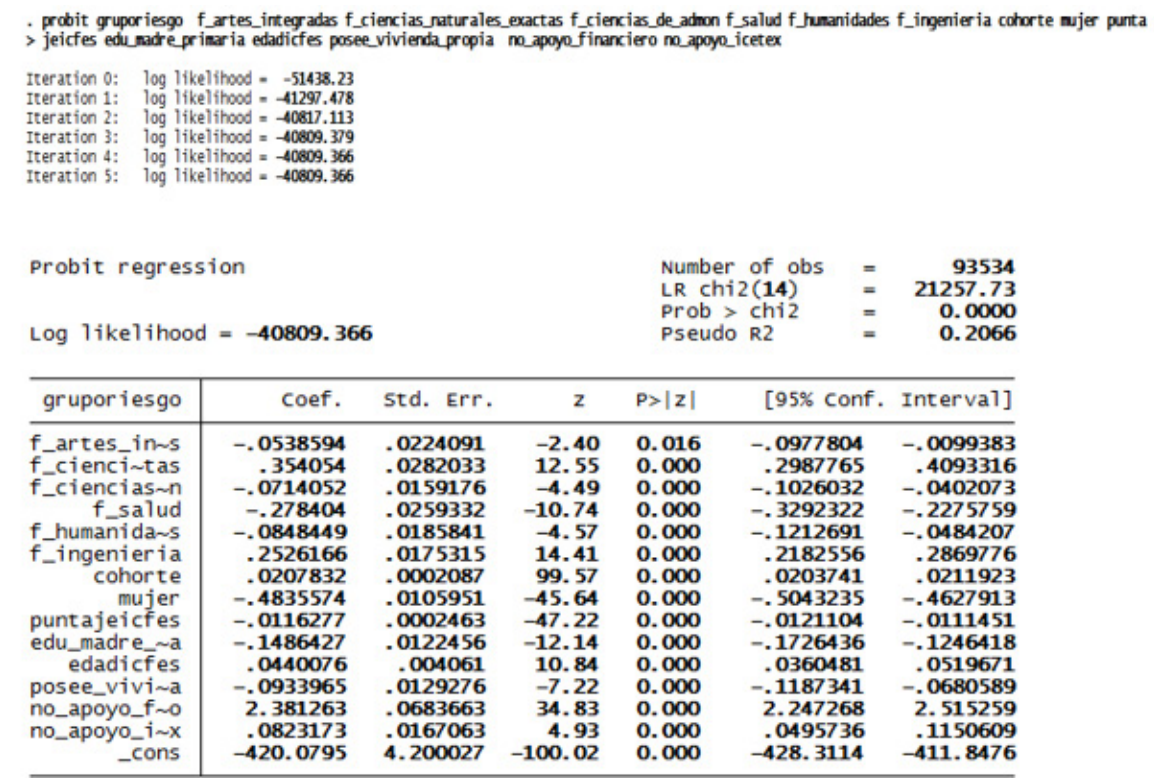

Anexo B.

Efector marginales de los determinantes probabilísticos del riesgo de deserción en la Universidad del Valle a partir del uso de modelos Probit. 2007-2017.

Marginal effects after probit

$=\operatorname{Pr}($ gruporiesgo) (predict)

\begin{tabular}{|c|c|c|c|c|c|c|c|}
\hline variable & $d y / d x$ & std. Err. & z & $\mathrm{P}>|\mathrm{z}|$ & $95 \%$ & C.I. & $\mathrm{x}$ \\
\hline & -.0133045 & .00541 & -2.46 & 0.014 & -.023917 & -.002 & 36 \\
\hline$f_{-} \mathrm{c}$ & .10288 & .00921 & 11.17 & & .084835 & .120941 & 39055 \\
\hline & -.0177484 & .0039 & -4.56 & 0. & -.025384 & -.010113 & $0^{\circ}$ \\
\hline $\bar{f}$ _salud* & -.0625829 & .0051 & -12.26 & 0.000 & -.072587 & -.052579 & .087 \\
\hline f_huma $\sim 5^{*}$ & -.020812 & .00443 & -4.70 & 0.000 & -.029485 & -.012139 & .1532 \\
\hline$f_{-i n g e} a^{*}$ & .0681847 & .00503 & 13.56 & 0.000 & .058327 & .078042 & .208 \\
\hline cohort & .0052459 & .00005 & 110.31 & & .005153 & .0 & 20 \\
\hline mujer* & -.1233775 & .00273 & -45.18 & 0.000 & .12873 & -.1 & .5258 \\
\hline puntaj s & -.0029349 & .00006 & -47.18 & 0.000 & -.003057 & -.0 & 75.8 \\
\hline edu_ma $a^{*}$ & -.0363392 & .0029 & -12.55 & 0.000 & -.042016 & -.030663 & .2793 \\
\hline edadic $\sim 5$ & .0111079 & .00102 & 10.84 & 0.000 & .009099 & .013117 & 16.92 \\
\hline osee_ a* & -.0242867 & .00346 & -7.01 & 0.000 & -.031073 & -.0175 & .839438 \\
\hline _ар & .1870659 & .00155 & 120.75 & 0.000 & .184029 & 190102 & .970567 \\
\hline no_apo $\sim X^{*}$ & .0201346 & .00395 & 5.09 & 0.000 & .012385 & .027884 & .892381 \\
\hline
\end{tabular}

(*) $d y / d x$ is for discrete change of dummy variable from 0 to 1

Anexo C.

Test de correcta clasificación de los determinantes probabilísticos del riesgo de

deserción en la Universidad del Valle a partir del uso de modelos Probit. 2007-2017.

. estat class, cutoff $(0.27)$

Probit model for gruporiesgo

\begin{tabular}{|c|c|c|c|}
\hline classified & D & $\sim D$ & Total \\
\hline+ & $\begin{array}{r}16146 \\
6210\end{array}$ & $\begin{array}{l}20161 \\
51017\end{array}$ & $\begin{array}{l}36307 \\
57227\end{array}$ \\
\hline Total & 22356 & 71178 & 93534 \\
\hline
\end{tabular}

Classified + if predicted $\operatorname{Pr}(D)>=.27$
True D defined as gruporiesgo $!=0$

\begin{tabular}{lll}
\hline Sensitivity & $\operatorname{Pr}(+\mid D)$ & $\mathbf{7 2 . 2 2 \%}$ \\
Specificity & $\operatorname{Pr}(-\mid \sim D)$ & $\mathbf{7 1 . 6 8 \%}$ \\
Positive predictive value & $\operatorname{Pr}(D \mid+)$ & $\mathbf{4 4 . 4 7 \%}$ \\
Negative predictive value & $\operatorname{Pr}(\sim D \mid-)$ & $\mathbf{8 9 . 1 5 \%}$ \\
\hline False + rate for true $\sim D$ & $\operatorname{Pr}(+\mid \sim D)$ & $\mathbf{2 8 . 3 2 \%}$ \\
False - rate for true $D$ & $\operatorname{Pr}(--\mid D)$ & $\mathbf{2 7 . 7 7 8 \%}$ \\
False + rate for classified + & $\operatorname{Pr}(\sim D \mid+)$ & $\mathbf{5 5 . 5 3 \%}$ \\
False - rate for classified - & $\operatorname{Pr}(D \mid-)$ & $\mathbf{1 0 . 8 5 \%}$ \\
\hline Correctly classified & & $\mathbf{7 1 . 8 1 \%}$ \\
\hline
\end{tabular}


Anexo D.

Determinantes probabilísticos del riesgo de deserción en la Universidad de Nariño a partir del uso de modelos Probit. 2007 - 2017.

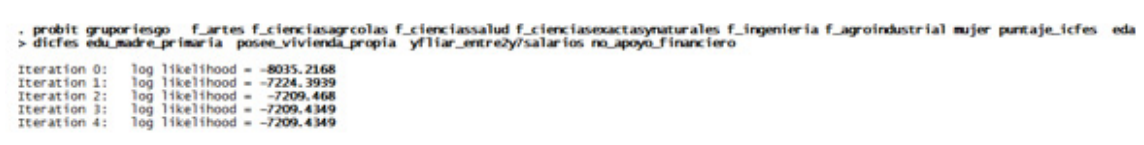

\begin{tabular}{|c|c|c|c|c|c|c|}
\hline \multicolumn{4}{|c|}{ Probit regression } & \multicolumn{2}{|c|}{$\begin{array}{l}\text { Number of obs } \\
\text { LR chi2 (13) } \\
\text { Prob }>\text { chi2 } \\
\text { Pseudo R2 }\end{array}$} & $\begin{array}{r}17055 \\
1651.56 \\
0.0000 \\
0.1028\end{array}$ \\
\hline gruporiesgo & coef. & Std. Err. & $\mathrm{z}$ & $P>|z|$ & [95\% conf & Interval] \\
\hline$a r$ & .190062 & .0322967 & & & .1 & \\
\hline 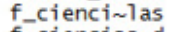 & & & & & & \\
\hline & & & & &.- & -.2 \\
\hline & & & & & & \\
\hline & & & & & & \\
\hline f_a & & & & & & \\
\hline 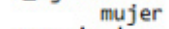 & & & & & & \\
\hline & 8 & & & & & -.0 \\
\hline & & & & & & .0 \\
\hline & 9435 & & & & & - . \\
\hline & -1246 & & & & -.1 & -.0 \\
\hline yf & & & & & & \\
\hline & & & & & & 92699 \\
\hline & 1.839918 & .2332035 & & 0.000 & -2.296989 & -1.38284 \\
\hline
\end{tabular}

Anexo E.

Efector marginales de los determinantes probabilísticos del riesgo de deserción en la Universidad de Nariño a partir del uso de modelos Probit. 2007 - 2017.

- $\mathrm{mfx}$

Marginal effects after probit

$$
\begin{aligned}
\mathrm{y} & =\operatorname{Pr}(\mathrm{grupor} i \mathrm{esgo}) \text { (predict) } \\
& =.15296094
\end{aligned}
$$

\begin{tabular}{|c|c|c|c|c|c|c|c|}
\hline variable & $\mathrm{dy} / \mathrm{dx}$ & Std. Err. & $z$ & $P>|z|$ & $95 \%$ & C.I. & $\mathrm{x}$ \\
\hline & 77672 & .00859 & ? & 0 & .030932 & .064 & בת \\
\hline & & & & & 963 & .149 & \\
\hline & -.0 & & -6. & & -.101596 & & \\
\hline$=0$ & .13 & & & & .106731 & & \\
\hline & & & 13. & & & & \\
\hline agr & & & & & .087014 & & \\
\hline mujer & -.09 & & & & 279 & & \\
\hline ntaj s & -.002541 & .00012 & & & -.00 & & \\
\hline $\operatorname{adic} \sim$ & .0110289 & .00271 & 4.07 & 0. & .005716 & .01 & 17.0277 \\
\hline U_ma & -.0222466 & .00622 & -3.57 & 0.000 & -.034445 & $-.01 c$ & . 482791 \\
\hline see & -.0300396 & .00637 & -4.72 & 0.000 & -.042519 & -.01756 & .658575 \\
\hline & .10 & .006 & 16 & $0 . c$ & .090234 & .113874 & .498036 \\
\hline & .1153642 & .00985 & 11.72 & 0.000 & .096064 & . 134665 & . 973028 \\
\hline
\end{tabular}

(*) $d y / d x$ is for discrete change of dummy variable from 0 to 1

Anexo F.

Test de correcta clasificación de los determinantes probabilísticos del riesgo de deserción en la Universidad del Valle a partir del uso de modelos Probit. 2007 - 2017.

. estat class, cutoff $(0.19)$

Probit model for gruporiesgo

\begin{tabular}{|c|c|c|c|}
\hline & & & \\
\hline classified & D & $\sim D$ & Total \\
\hline+ & $\begin{array}{l}1985 \\
1082\end{array}$ & $\begin{array}{l}4613 \\
9375\end{array}$ & $\begin{array}{r}6598 \\
10457\end{array}$ \\
\hline ta & 3067 & 13988 & 17055 \\
\hline
\end{tabular}

Classified + if predicted $\operatorname{Pr}(D)>=.19$

\begin{tabular}{lll}
\hline Sensitivity & $\operatorname{Pr}(+\mid$ D) & $\mathbf{6 4 . 7 2 \%}$ \\
Specificity & $\operatorname{Pr}(-\mid \sim D)$ & $\mathbf{6 7 . 0 2 \%}$ \\
Positive predictive value & $\operatorname{Pr}(D \mid+)$ & $\mathbf{3 0 . 0 8 \%}$ \\
Negative predictive value & $\operatorname{Pr}(\sim D \mid-)$ & $\mathbf{8 9 . 6 5 \%}$ \\
\hline False + rate for true $\sim D$ & $\operatorname{Pr}(+\mid \sim D)$ & $\mathbf{3 2 . 9 8 \%}$ \\
False - rate for true D & $\operatorname{Pr}(-\mid D)$ & $\mathbf{3 5 . 2 8 \%}$ \\
False + rate for classified + & $\operatorname{Pr}(\sim D \mid+)$ & $\mathbf{6 9 . 9 2 \%}$ \\
False - rate for classified - & $\operatorname{Pr}(D \mid-)$ & $\mathbf{1 0 . 3 5 \%}$ \\
\hline correctly classified & & $\mathbf{6 6 . 6 1 \%}$ \\
\hline
\end{tabular}

